\title{
Cell Model for the Scale-up of Fluid Dynamics and Mass Transfer in Random Packed Columns Affected by Maldistribution
}

\author{
Jost Brinkmann ${ }^{1}$, Amelie Merkel $^{1}$, Dominik Plate ${ }^{1}$, and Marcus Gruenewald ${ }^{1}$ \\ ${ }^{1}$ Ruhr University Bochum
}

April 19, 2021

\begin{abstract}
The characterization of fluid dynamics and mass transfer is often influenced by phase maldistribution. An existing cell model approach for fluid distribution is extended for gas distribution and mass transfer. The model differentiates wall and bulk zones, allowing a detailed evaluation of local fluid dynamics and mass transfer, which is based on a state-of-the-art literature correlation. Distribution parameters are determined experimentally. The model allows scale-up by considering the phase distribution at constant computational effort. A scale-up strategy to adapt the distribution parameters to different geometries is presented. Results for fluid dynamics and liquid distribution are in good agreement with experimental data of columns with diameters of $0.288,0.5$ and $0.6 \mathrm{~m}$. Mass transfer is in good qualitative agreement with own experiments in a 0.288 diameter column. While maldistribution consequently reduces the gas side mass transfer, the reduced liquid bulk flow weakens the effect of liquid side mass transfer.
\end{abstract}

\section{Hosted file}

MainDocumentWord.pdf available at https://authorea.com/users/408811/articles/518678-cellmodel-for-the-scale-up-of-fluid-dynamics-and-mass-transfer-in-random-packed-columnsaffected-by-maldistribution

A)

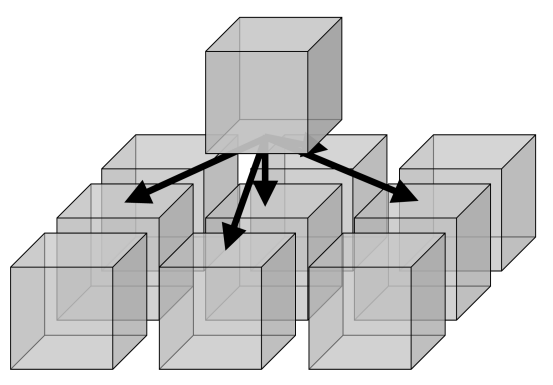

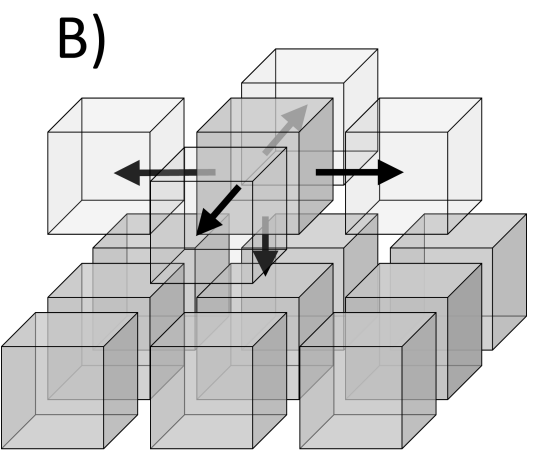




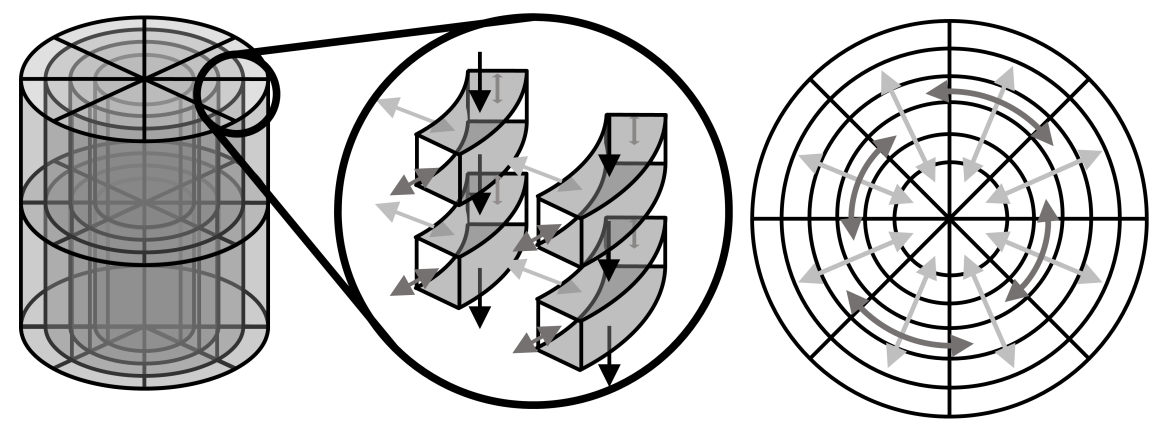

radial $(\leftrightarrow)$, tangential $(\leftrightarrow)$ \& vertical $(\rightarrow)$ fluid distribution

A)

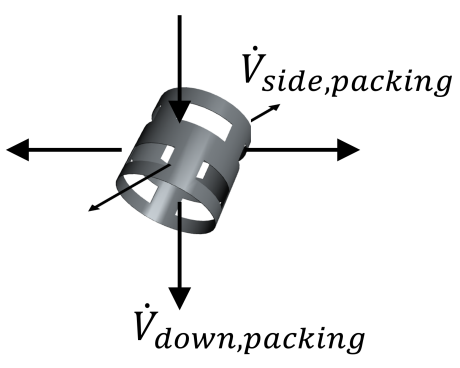

Bulk cell

Fluid dynamics: $\mathrm{H}_{\mathrm{L}}, \Delta \mathrm{p} \cdot \mathrm{H}^{-1}$

$\Rightarrow$ Billet \& Schultes

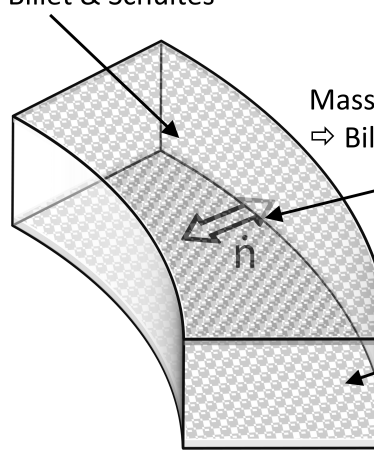

B)

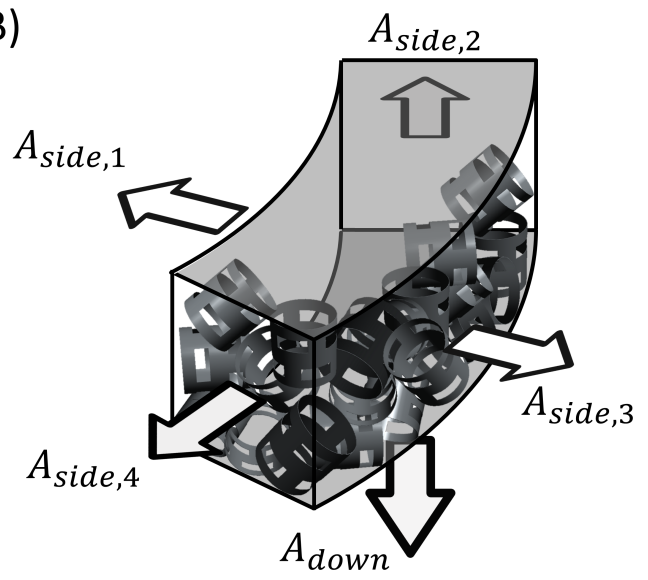

Wall cell

Hold-up: $\mathrm{H}_{\mathrm{L}} \quad$ Phase interface: $\mathrm{a}_{\text {Wall }}$

$\Rightarrow$ Film theory $\quad \Rightarrow$ Film theory

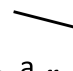

Mass transfer: $\beta_{L}, \beta_{G}, a_{\text {eff }}$

Billet \& Schultes

Loading \& flooding: $\mathrm{F}_{\mathrm{V}, \mathrm{S}}, \mathrm{F}_{\mathrm{V}, \mathrm{Fl}}$

$\Rightarrow$ Billet \& Schultes

Pressure drop: $\Delta \mathrm{p} \cdot \mathrm{H}^{-1}$

$\Rightarrow$ Billet und Schultes 

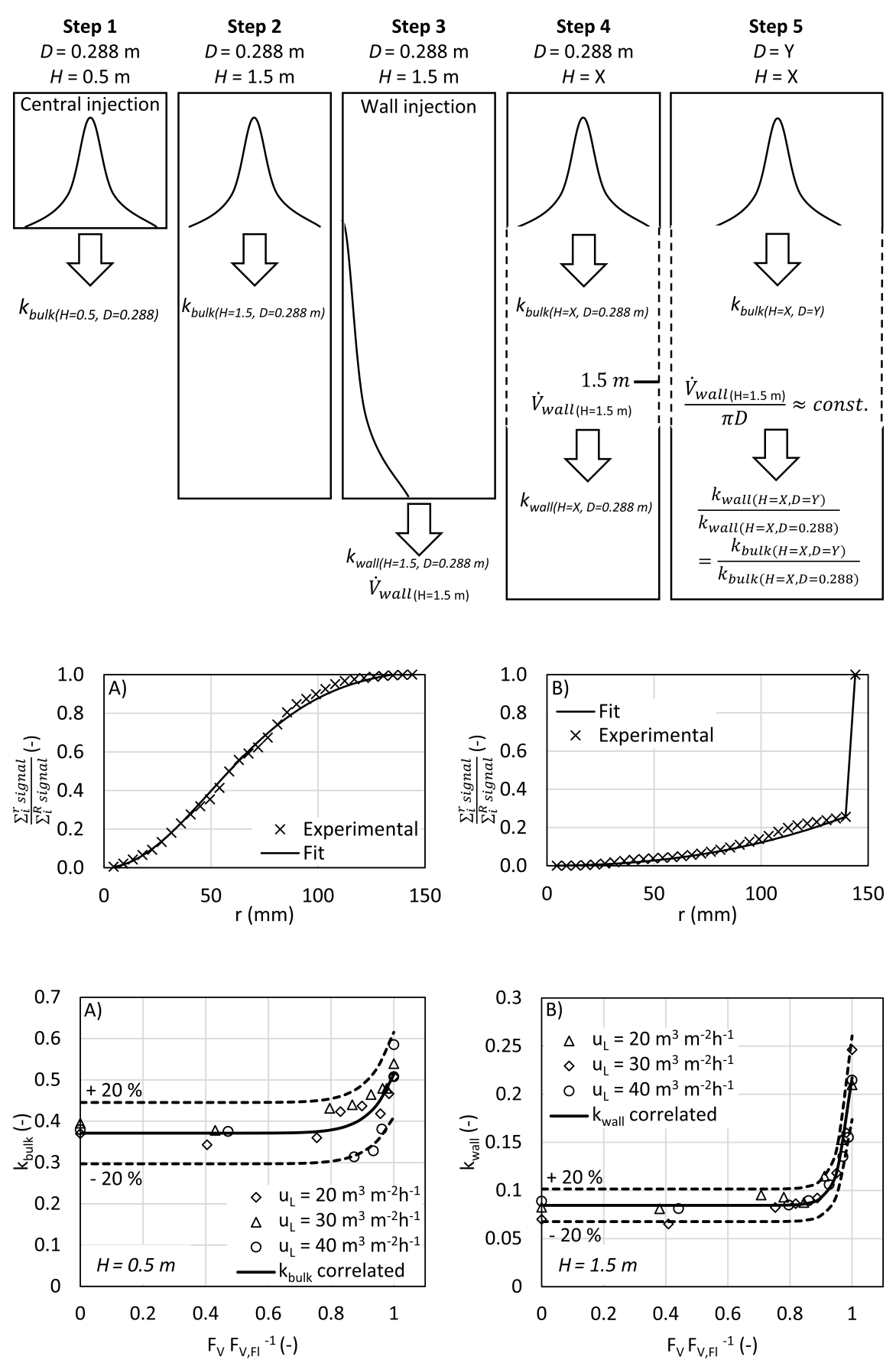

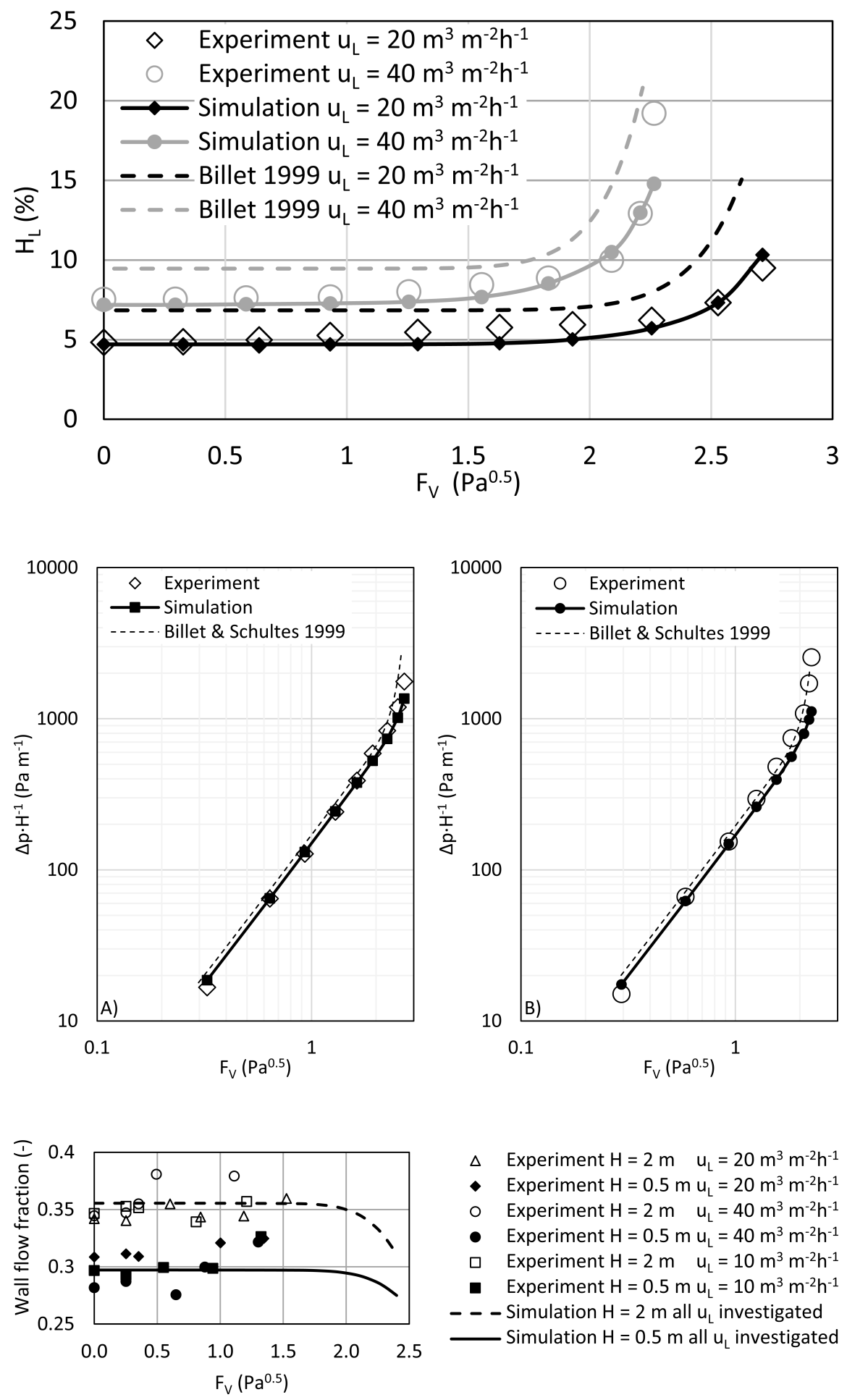

$\triangle \quad$ Experiment $\mathrm{H}=2 \mathrm{~m} \quad \mathrm{u}_{\mathrm{L}}=20 \mathrm{~m}^{3} \mathrm{~m}^{-2} \mathrm{~h}^{-1}$

- Experiment $\mathrm{H}=0.5 \mathrm{~m} \mathrm{u}_{\mathrm{L}}=20 \mathrm{~m}^{3} \mathrm{~m}^{-2} \mathrm{~h}^{-1}$

- Experiment $\mathrm{H}=2 \mathrm{~m} \quad \mathrm{u}_{\mathrm{L}}=40 \mathrm{~m}^{3} \mathrm{~m}^{-2} \mathrm{~h}^{-1}$

- Experiment $\mathrm{H}=0.5 \mathrm{~m} \mathrm{u}_{\mathrm{L}}=40 \mathrm{~m}^{3} \mathrm{~m}^{-2} \mathrm{~h}^{-1}$

ㅁ Experiment $\mathrm{H}=2 \mathrm{~m} \quad \mathrm{u}_{\mathrm{L}}=10 \mathrm{~m}^{3} \mathrm{~m}^{-2} \mathrm{~h}^{-1}$

- Experiment $\mathrm{H}=0.5 \mathrm{~m} \mathrm{u}_{\mathrm{L}}=10 \mathrm{~m}^{3} \mathrm{~m}^{-2} \mathrm{~h}^{-1}$

- - - Simulation $\mathrm{H}=2 \mathrm{~m}$ all $\mathrm{u}_{\mathrm{L}}$ investigated

Simulation $\mathrm{H}=0.5 \mathrm{~m}$ all $\mathrm{u}_{\mathrm{L}}$ investigated 

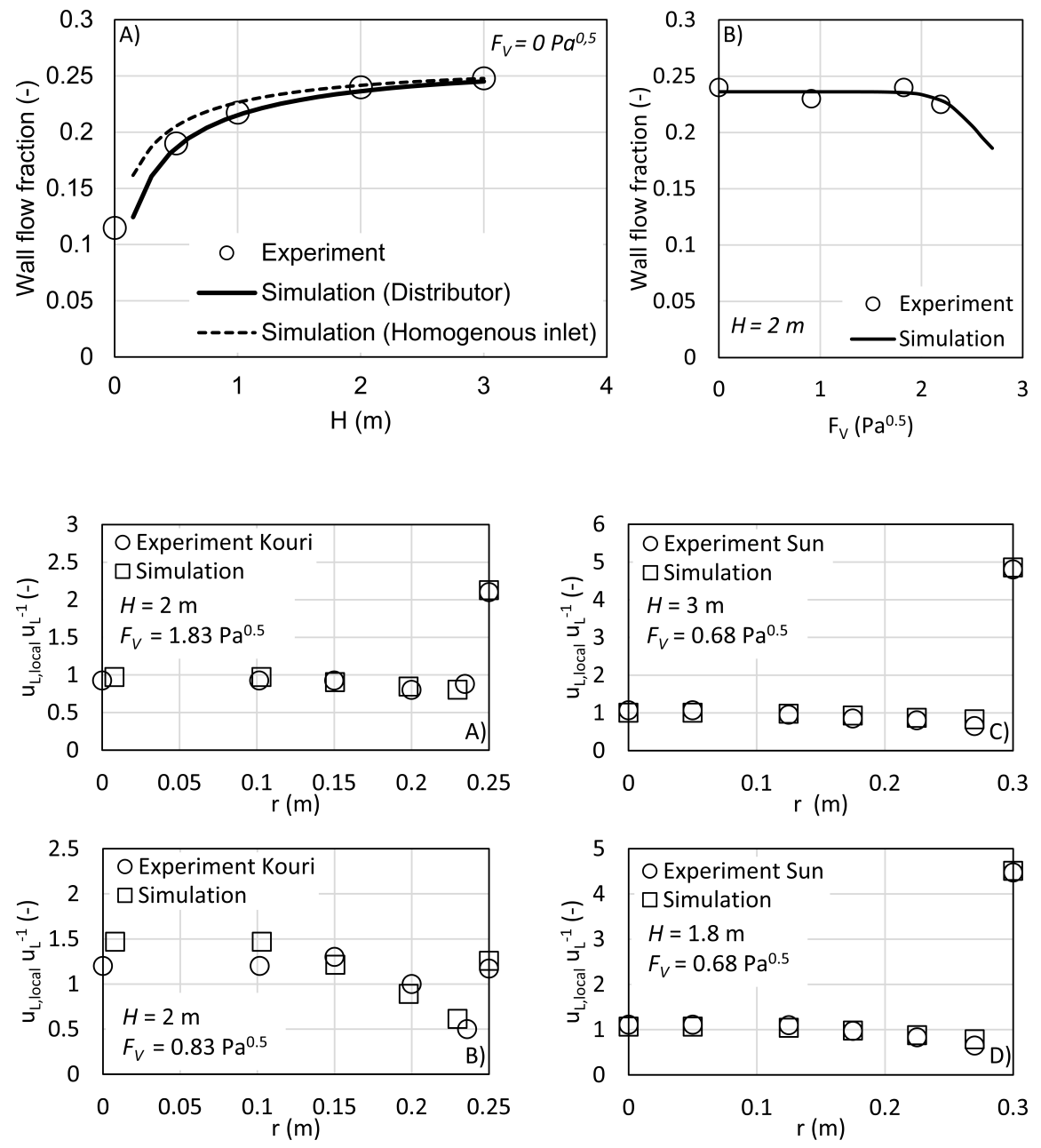

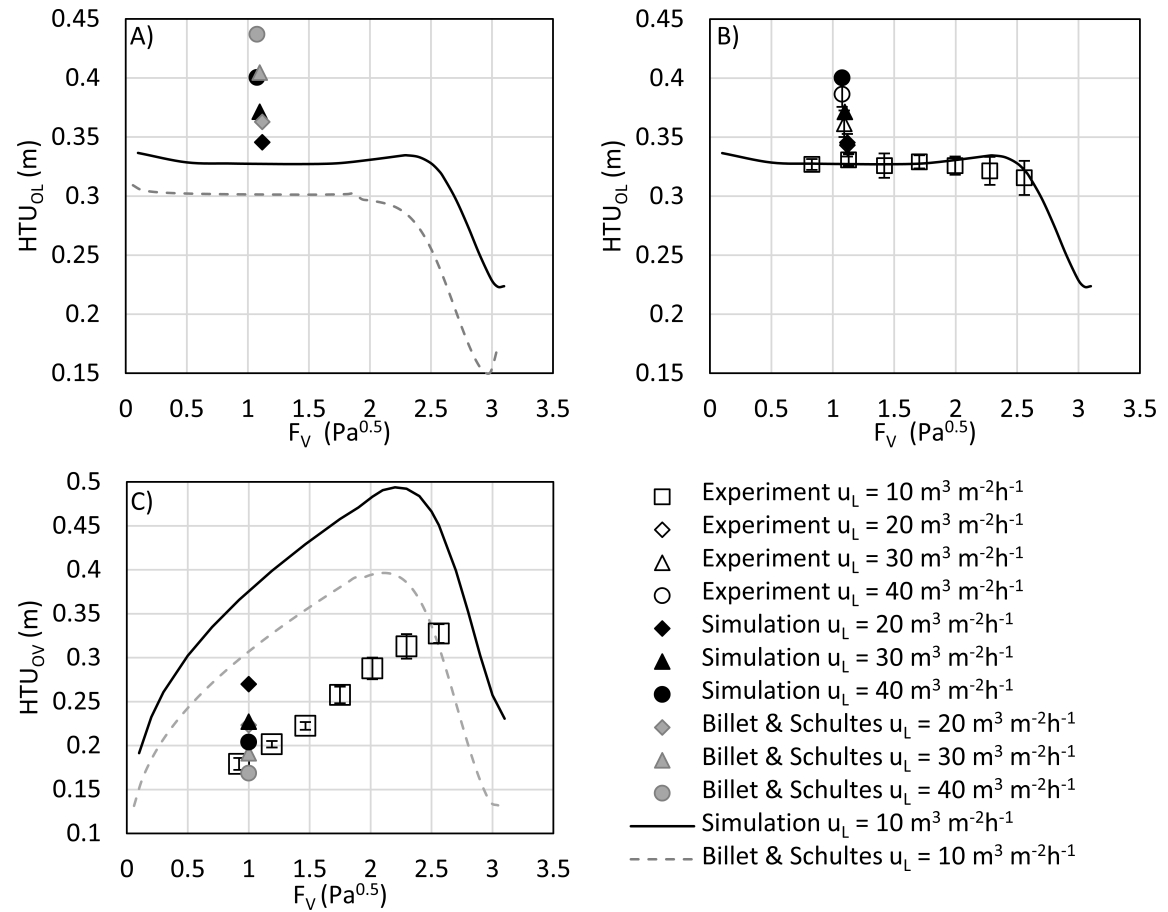

$\square$ Experiment $u_{L}=10 \mathrm{~m}^{3} \mathrm{~m}^{-2} \mathrm{~h}^{-1}$

$\diamond$ Experiment $\mathrm{u}_{\mathrm{L}}=20 \mathrm{~m}^{3} \mathrm{~m}^{-2} \mathrm{~h}^{-1}$

$\triangle$ Experiment $u_{L}=30 \mathrm{~m}^{3} \mathrm{~m}^{-2} \mathrm{~h}^{-1}$

- Experiment $u_{1}=40 \mathrm{~m}^{3} \mathrm{~m}^{-2} \mathrm{~h}^{-1}$

- Simulation $u_{L}=20 \mathrm{~m}^{3} \mathrm{~m}^{-2} \mathrm{~h}^{-1}$

- Simulation $u_{1}=30 \mathrm{~m}^{3} \mathrm{~m}^{-2} \mathrm{~h}^{-1}$

- Simulation $\mathrm{u}_{\mathrm{L}}=40 \mathrm{~m}^{3} \mathrm{~m}^{-2} \mathrm{~h}^{-1}$

$\triangle$ Billet \& Schultes $u_{\mathrm{L}}=20 \mathrm{~m}^{3} \mathrm{~m}^{-2} \mathrm{~h}^{-1}$

$\triangle \quad$ Billet \& Schultes $u_{L}=30 m^{3} m^{-2} h^{-1}$

- Billet \& Schultes $u_{L}=40 \mathrm{~m}^{3} \mathrm{~m}^{-2} \mathrm{~h}^{-1}$ Simulation $u_{\mathrm{L}}=10 \mathrm{~m}^{3} \mathrm{~m}^{-2} \mathrm{~h}^{-1}$

- - - - Billet \& Schultes $u_{\llcorner}=10 \mathrm{~m}^{3} \mathrm{~m}^{-2} \mathrm{~h}^{-1}$ 